\title{
A SPECIAL DETERMINANT
}

\section{CARLITZ}

Let $p$ be a prime and let $R(r)$ denote the least non-negative residue of $r(\bmod p)$. The properties of Maillet's determinant (for references see [2]) $D_{p}=\left|R\left(r s^{\prime}\right)\right|(r, s=1, \cdots,(p-1) / 2)$ where $s s^{\prime} \equiv 1(\bmod$ $p)$, suggest that it may be of interest to discuss the determinant

$$
\Delta_{k}=\left|R\left((r-s)^{k}\right)\right| \quad(r, s=0, \cdots, p-1 ; 1 \leqq k \leqq p-1) .
$$

Clearly $\Delta_{k}$ is a circulant. Consequently

$$
\Delta_{k}=\sum_{r=1}^{p-1} R\left(\boldsymbol{r}^{k}\right) \cdot \prod_{i=1}^{p-1} \sum_{r=1}^{p-1} R\left(\boldsymbol{r}^{k}\right) \epsilon^{r s}, \quad \text { where } \epsilon=e^{2 \pi i / p}
$$

Now by the binomial theorem

$$
\begin{aligned}
(1-\epsilon)^{p-1-k} & \equiv \sum_{r=0}^{p-1-k} \frac{(k+1) \cdots(k+r)}{r !} \epsilon^{r} \\
& \equiv \sum_{r=0}^{p-1-k} \frac{(r+1) \cdots(r+k)}{k !} \epsilon^{r} \quad(\bmod p) .
\end{aligned}
$$

Next recall that (see for example [3, p. 207])

$$
x^{k}=\sum_{s=1}^{k} a_{k s}\left(\begin{array}{c}
x+s-1 \\
k
\end{array}\right) \quad\left(a_{k s}=a_{k, k-s+1}, k \geqq 1\right),
$$

where the $a_{k s}$ (Eulerian coefficients) are positive integers; clearly (4) implies

$$
\sum_{i=1}^{k} a_{k s}=k !
$$

Then using (3) and (4) we get

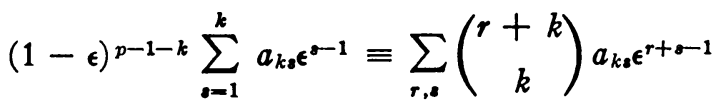

$$
\begin{aligned}
& \equiv \sum_{t=1}^{p-1} \epsilon^{t-1} \sum_{t=1}^{k} a_{k \bullet}\left(\begin{array}{c}
t-s+k \\
k
\end{array}\right) \\
& \equiv \sum_{t=1}^{p-1} t^{k} \epsilon^{t-1} \quad(\bmod p) \text {. }
\end{aligned}
$$

Received by the editors May 25, 1954. 
Also it is clear from (5) that

$$
\sum_{s=1}^{k} a_{k s} \epsilon^{-1} \equiv \sum_{s=1}^{k} a_{k s} \equiv k ! \quad(\bmod 1-\epsilon) .
$$

Thus it follows from (6) and (7) that the number

$$
\alpha=\sum_{t=1}^{p-1} t^{k} \epsilon^{t-1}
$$

is divisible by $(1-\epsilon)^{p-1-k}$ and not by $(1-\epsilon)^{p-k}$. We recall that in the cyclotomic field generated by $\epsilon$ we have the prime ideal factorization

$$
(p)=(1-\epsilon)^{p-1} \text {. }
$$

Now in the double product in the right member of (2), the sum

$$
\sum_{r=1}^{p-1} R\left(r^{k}\right) \epsilon^{r s} \equiv \epsilon^{s} \sum_{r=1}^{p-1} r^{k} \epsilon^{s(r-1)}
$$

and is therefore divisible by exactly $(1-\epsilon)^{p-1-k}$. More precisely by (7)

$$
\sum_{r=1}^{p-1} R\left(r^{k}\right) \epsilon^{r s} \equiv \epsilon^{s} k !(1-\epsilon)^{p-1-k} \quad\left(\bmod (1-\epsilon)^{p-k}\right)
$$

and therefore

$$
(1-\epsilon)^{-p+1+k} \sum_{r=1}^{p-1} R\left(r^{k}\right) \epsilon^{r s} \equiv \epsilon^{8} k ! \quad(\bmod 1-\epsilon) .
$$

Multiplying together these congruences we get

$$
p^{-p+1+k} \prod_{s=1}^{p-1} \sum_{r=1}^{p-1} R\left(r^{k}\right) \epsilon^{r s} \equiv(k !)^{p-1} \equiv 1 \quad(\bmod 1-\epsilon) .
$$

Since the left number is a rational integer, $(8)$ holds $(\bmod p)$. Thus substituting in (2) it is clear that for $k<p-1$

$$
\Delta_{k} \equiv p^{p-1-k} \sum_{r=1}^{p-1} R\left(r^{k}\right) \quad\left(\bmod p^{p-k+1}\right) .
$$

Put

$$
S_{k}=\sum_{r=1}^{p-1} R\left(r^{k}\right)
$$

since $p \mid S_{k}, p^{2} \nmid S_{k}$ for $1 \leqq k<p-1$, it follows from (9) that

$$
p^{p-k} \mid \Delta_{k}, p^{p-k+1} \nmid \Delta_{k} \quad(1 \leqq k<p-1) .
$$


To get a more precise result, note first that if $a=(k, p-1)$, then $S_{k}=S_{a}$. Put $p-1=a b$; then in the first place

$$
S_{a}=p(p-1) / 2 \quad \text { ( } b \text { even). }
$$

For $b$ odd, on the other hand, we may prove by the method used in [1] that

$$
S_{a} \equiv-\frac{p}{2}+p \sum_{u} \frac{B_{b u+1}}{b u+1} \quad\left(\bmod p^{2}\right),
$$

where $B_{m}$ denotes a Bernoulli number in the even suffix notation, and the summation is over $u=1,3, \cdots, a-1$.

We remark that for $k=p-1$ we have the easily verified formula

$$
\Delta_{p-1}=\left|1-\delta_{r s}\right|=p-1,
$$

where $\delta_{r s}$ is the Kronecker delta. Also for $k=1$ we have the exact result

$$
\Delta_{1}=(p-1) p^{p-1} / 2 .
$$

It follows from (2) and (8) that $\Delta_{k}$ never vanishes.

\section{REFERENCES}

1. L. Carlitz, The first factor of the class number of a cyclic field, Canadian Journal of Mathematics vol. 6 (1954) pp. 23-26.

2. L. Carlitz and F. R. Olson, Maillet's determinant, Proc. Amer. Math. Soc. vol. 6 (1955) pp. 265-269.

3. J. Worpitzky, Studien über die Bernoullischen und Eulerschen Zahlen, J. Reine Angew. Math. vol. 94 (1883) pp. 202-322.

DURE UNIVERSITY 